\title{
Preoperative Prognostic Value of MRI Findings in 108 Patients with Idiopathic Normal Pressure Hydrocephalus
}

\author{
J. Virhammar, K. Laurell, K.G. Cesarini, and E.-M. Larsson
}

\begin{abstract}
BACKGROUND AND PURPOSE: MR imaging is used in the diagnostic evaluation of patients with idiopathic normal pressure hydrocephalus. The aim of this study was to describe the prevalence of several imaging features and their prognostic use in the selection of shunt candidates with idiopathic normal pressure hydrocephalus.
\end{abstract}

MATERIALS AND METHODS: Preoperative MR imaging scans of the brain were retrospectively evaluated in 108 patients with idiopathic normal pressure hydrocephalus who had undergone a standardized, clinical evaluation before and 12 months after shunt surgery. The MR imaging features investigated were the Evans index, callosal angle, narrow sulci at the high convexity, dilation of the Sylvian fissure, diameters of the third ventricle and temporal horns, disproportionately enlarged subarachnoid space hydrocephalus, flow void through the aqueduct, focal bulging of the roof of the lateral ventricles, deep white matter hyperintensities, periventricular hyperintensities, and focal widening of sulci and aqueductal stenosis.

RESULTS: In logistic regression models, with shunt outcome as a dependent variable, the ORs for the independent variables, callosal angle, disproportionately enlarged subarachnoid space hydrocephalus, and temporal horns, were significant $(P<.05)$, both in univariate analyses and when adjusted for age, sex, and previous stroke.

CONCLUSIONS: A small callosal angle, wide temporal horns, and occurrence of disproportionately enlarged subarachnoid space hydrocephalus are common in patients with idiopathic normal pressure hydrocephalus and were significant predictors of a positive shunt outcome. These noninvasive and easily assessed radiologic markers could aid in the selection of candidates for shunt surgery.

ABBREVIATIONS: DESH = disproportionately enlarged subarachnoid space hydrocephalus; DWMH = deep white matter hyperintensities; ICC = intraclass correlation coefficient; $\mathrm{NPH}=$ idiopathic normal pressure hydrocephalus; $\mathrm{NPH}=$ normal pressure hydrocephalus; $\mathrm{PVH}=$ periventricular hyperintensities; $\mathrm{SINPHONI}=\mathrm{Study}$ of Idiopathic Normal Pressure Hydrocephalus on Neurological Improvement

\begin{abstract}
diopathic normal pressure hydrocephalus (iNPH) is a disease of the elderly population, with symptoms of balance and gait disturbances, dementia, and urinary incontinence; and in approximately 8 of 10 patients, the symptoms are reversible after shunt insertion. ${ }^{1,2}$ Several imaging methods have been used in the diagnosis and selection of shunt candidates, but the standard method at present is MR imaging. Even though advanced functional imaging methods are being considered, the clinical evaluation of patients with iNPH still often relies on morphologic findings.
\end{abstract}

However, whether morphologic findings on preoperative im-

Received March 30, 2014; accepted after revision May 23.

From the Departments of Neuroscience and Neurology (J.V., K.L.), Neuroscience and Neurosurgery (K.G.C.), and Radiology (E.-M.L.), Uppsala University, Uppsala, Sweden; and Department of Pharmacology and Clinical Neuroscience (K.L.), Östersund, Umeå University, Umeå, Sweden.

Please address correspondence to J. Virhammar, MD, PhD, Department of Neuroscience and Neurology, Uppsala University, Akademiska Sjukhuset, ing 85, 75185 Uppsala, Sweden; e-mail: johan.virhammar@neuro.uu.se; @johanvirhammar.

http://dx.doi.org/10.3174/ajnr.A4046 aging are of prognostic value is controversial, and there is no consensus regarding which MR imaging sequences and variables to evaluate in the selection of shunt candidates. In addition, it is not fully clear how the radiologic features are related to each other and to the symptoms. Previous studies have often involved a limited number of patients and a mixed sample of both idiopathic normal pressure hydrocephalus and normal pressure hydrocephalus (NPH) secondary to a brain insult.

Therefore, we investigated 108 patients who had undergone shunt surgery for iNPH, with the aim of describing the occurrence and prognostic value of 13 different radiologic variables and the relation between MR imaging findings and clinical symptoms.

\section{MATERIALS AND METHODS Materials}

The sample consisted of 108 patients with iNPH who underwent shunt surgery between 2006 and 2010 at the authors' hospital. Median age at the time of surgery was 74 years (range, 54-88 years); 58 (54\%) were men and 50 (46\%) were women. Inclusion 
criteria were a preoperative MR imaging examination of the brain and a clinical evaluation before and at 12 months after shunt surgery. All patients had ventricular enlargement and gradually evolving symptoms, including a gait disturbance with or without cognitive decline or urinary incontinence. Patients with secondary NPH were excluded. During the follow-up period, 29 patients had various types of shunt complications and 5 patients had complications related to comorbidity. The sample is described in detail in a previous study. ${ }^{3}$

\section{Clinical Evaluation}

Patients referred to Uppsala University Hospital because of clinical and radiologic symptoms of NPH were evaluated prospectively before surgery and at follow-up 12 months postoperatively by a multidisciplinary team specialized in hydrocephalus. A neurologist performed a clinical and neurologic evaluation, including a medical history covering intracranial hemorrhage, meningitis, or trauma or other causes of secondary hydrocephalus. All patients were assessed according to a prospective, standardized protocol developed to follow symptoms with time and to measure the outcome after shunt surgery. The cognitive function was tested with the Mini-Mental State Examination; urinary symptoms, with 1 ordinal continence scale ${ }^{4}$; motor function, with 1 ordinal balance scale ${ }^{4}$ and 1 ordinal gait scale ${ }^{4}$; and we also used 3 tests in which time and the number of steps were measured. The latter included walking $10 \mathrm{~m}$ at a self-chosen speed, a Timed $\mathrm{Up}$ and Go Test, and walking backward $3 \mathrm{~m}$.

A shunt responder was defined as a patient improved in any of the following 3 criteria:

1) Motor function of $\geq 1$ level on the gait or balance scale or $\geq 20 \%$ reduction in the time or the number of steps in $\geq 50 \%$ of the 3 tests

2) Cognition $\geq 4$ levels in the Mini-Mental State Examination

3) Continence scale $\geq 1$ level and improvement in the MiniMental State Examination score of $\geq 2$ levels.

A dichotomous variable shunt outcome was created and used as a dependent variable in the logistic regression. The modified Rankin Scale was used as a measure of general handicap level.

\section{Imaging}

Forty-one (38\%) of the preoperative MR imaging examinations were performed at the authors' hospital, and 67 (62\%), at the referring hospitals. Ten (9\%) were performed on a 3T scanner; 75 $(70 \%)$, on a $1.5 \mathrm{~T}$ scanner; $15(14 \%)$, on a $1 \mathrm{~T}$ scanner; and $8(7 \%)$, on a $0.5 \mathrm{~T}$ scanner.

Evaluation of MR images was performed retrospectively, with the investigators blinded to the patients' clinical data. Multiplanar reconstruction of the images was used as described below. For continuous variables, 2 investigators analyzed 20 randomly selected patients, and intraclass correlation coefficients (ICCs) were calculated to obtain inter-rater reliability. For the ordinal and dichotomous variables, 2 investigators independently analyzed all patients; and to test reliability, Cohen $\kappa$ was calculated. In cases of discrepancy in the evaluation, the images were re-evaluated, and a consensus was reached.

The Evans index was measured on transverse images as the ratio between the maximum diameter of the frontal horns of the lateral ventricles and the maximum inner diameter of the skull in the same section (Fig 1A).

The callosal angle measured on MR imaging is the angle between the lateral ventricles on a coronal image through the posterior commissure, perpendicular to the anterior/posterior commissure plane (Fig 1B). ${ }^{5}$

Compression of the medial and/or high convexity cortex sulci (narrow sulci) was evaluated on coronal and transverse images. ${ }^{6}$ It was graded as the following: $0=$ normal or wider than normal, $1=$ slight compression, $2=$ definitive compression (Fig $1 C,-D)$.

Dilation of the Sylvian fissure was graded in 3 different ways. The first method was described by Kitagaki et $\mathrm{al}^{7}$ and later illustrated with coronal images by Hashimoto et al. ${ }^{8}$ We graded dilation as the following: $0=$ normal or narrow, $1=$ mildly moderately enlarged, 2 = highly enlarged. ${ }^{7,8}$ When we used this method, the reliability between the 2 investigators was not sufficient $(\kappa=$ 0.36 , Fig 2 and Table 1). Therefore, we created a new grading scale, the Sylvian fissure ordinal, an ordinal scale with 3 levels and grading still based on the images of Hashimoto et al but with a more precise definition of how to reconstruct the coronal images to attain a higher reproducibility. The coronal images used for the Sylvian fissure ordinal were reconstructed at the level of the central part of the brain stem and angulated along the brain stem (Fig $2 B$ ). In the third method, the height of the Sylvian fissure was measured quantitatively on a sagittal image located at the midpoint between the skull and the insular cortex. The height was measured in millimeters in 5 different locations perpendicular to the direction of the Sylvian fissure. The median value of the 5 locations was calculated for each side, and the average of right and left was recorded.

Disproportionately enlarged subarachnoid space hydrocephalus (DESH) refers to a communicating hydrocephalus with enlarged ventricles and a disproportionate distribution of the CSF between the inferior and superior subarachnoid spaces. ${ }^{8}$ It was graded as present if both narrow sulci at the high convexity and the Sylvian fissure ordinal were graded as $\geq 1$ (Fig 1D).

Flow void phenomenon through the aqueduct and fourth ventricle (flow void) was graded by a modified version of the ordinal scale described by Algin et al. ${ }^{9}$ It was graded as follows: $0=$ no flow in the aqueduct, 1 = flow void only in the aqueduct, 2 = flow void in the aqueduct and the upper half of the fourth ventricle, 3 = flow void that extends to the caudal part of the fourth ventricle. It was graded on T2-weighted sagittal images without flow compensation. Only examinations from 1 scanner (Avanto 1.5T; Siemens, Erlangen, Germany) were included to avoid scanner differences with regard to flow compensation $(n=36$, Fig $1 E)$.

Focal bulging of the roof of the lateral ventricles was evaluated on sagittal images and was graded as present or not present (Fig 3).

The diameter of the third ventricle was measured in millimeters on transverse T1-weighted images in the center of the ventricle in the anteroposterior direction but in the widest part in the inferior-superior direction (Fig $1 F$ ). The maximal diameter of the temporal horns was measured in millimeters on each side on transverse images, and the average of left and right was calculated (Fig 1G).

Deep white matter hyperintensities (DWMH) were evaluated 

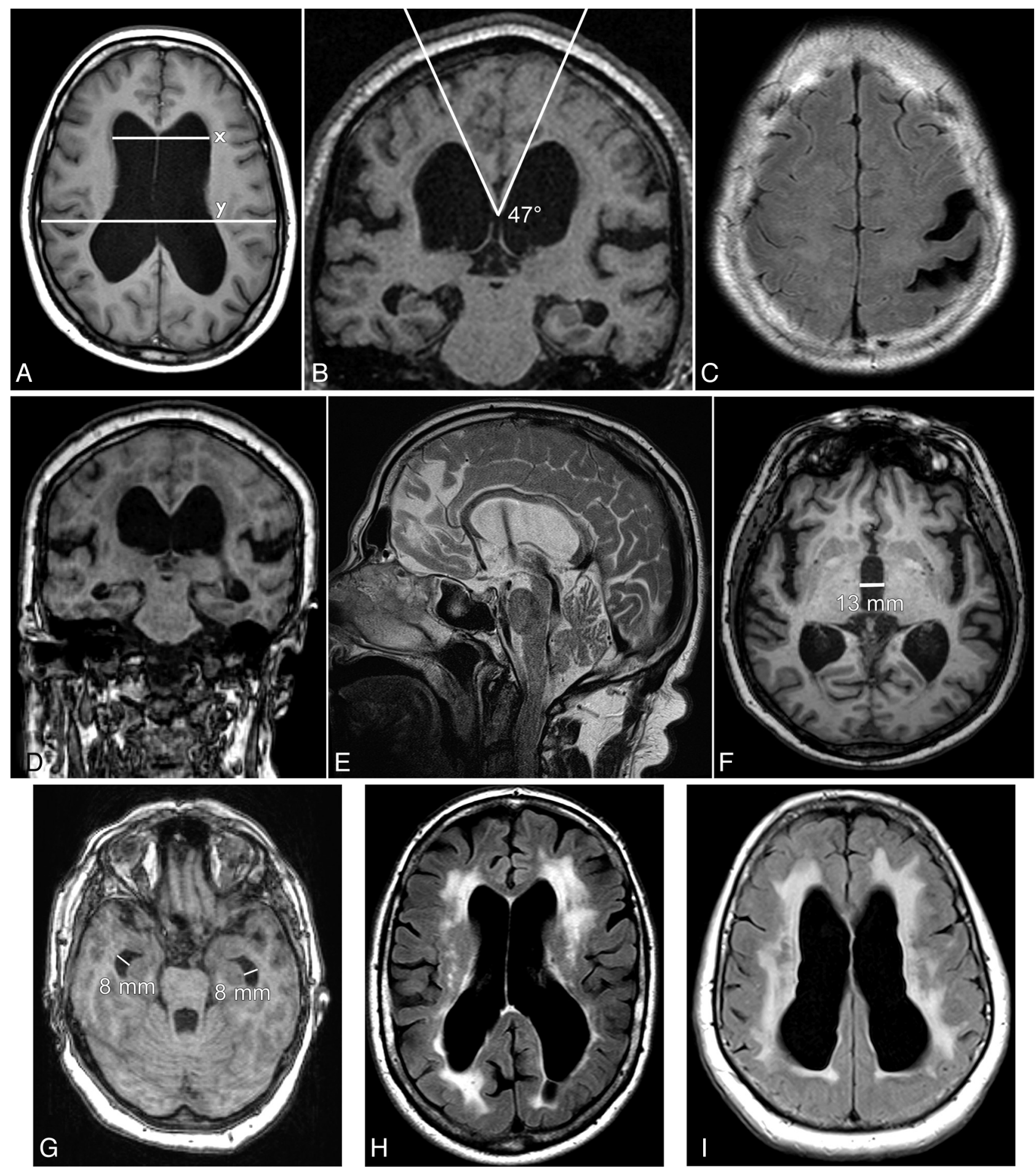

FIG 1. Nine different patients with iNPH. A, Evans index $=\mathrm{x} / \mathrm{y} . B$, Callosal angle. $C$, Narrow medial sulci and 2 focally dilated sulci on the left side. $D, D E S H . E$, A flow void in the aqueduct and fourth ventricle graded as 2 . In addition, a flow void in the foramina of Monro. $F$, Large diameter of the third ventricle. G, Dilated temporal horns. H, DWMH graded as 3 in a patient who also has PVH. I, PVH graded as 2.

on T2 FLAIR images by the ordinal scale described by Fazekas et $\mathrm{al}^{10}$ as follows: $0=$ no lesions, $1=$ punctate foci, $2=$ beginning confluence of foci, 3 = large confluent areas (Fig $1 H$ ). All clearly visible single lesions were graded as punctate foci.

Periventricular hyperintensities (PVH) along and always in contact with the frontal and parietal portions of the lateral ventricles were evaluated on T2 FLAIR images. They were graded as follows: $0=$ normal (including a "pencil-thin lining" along the ventricular wall and small caps around the frontal horns), $1=$ increased PVH, 2 = irregular large symmetric hyperintensities extending out into the deep white matter and, on at least 2 locations, all the way from the ventricles to the cortex (Fig $1 I) .{ }^{10}$

Accumulation of CSF in focally enlarged sulci, previously called "transport sulci," was evaluated as present or not present (Fig 1C). ${ }^{11,12}$

Focal narrowing of the aqueduct with suspicion of aqueductal AJNR Am J Neuroradiol 35:2311-18 Dec 2014 www.ajnr.org 

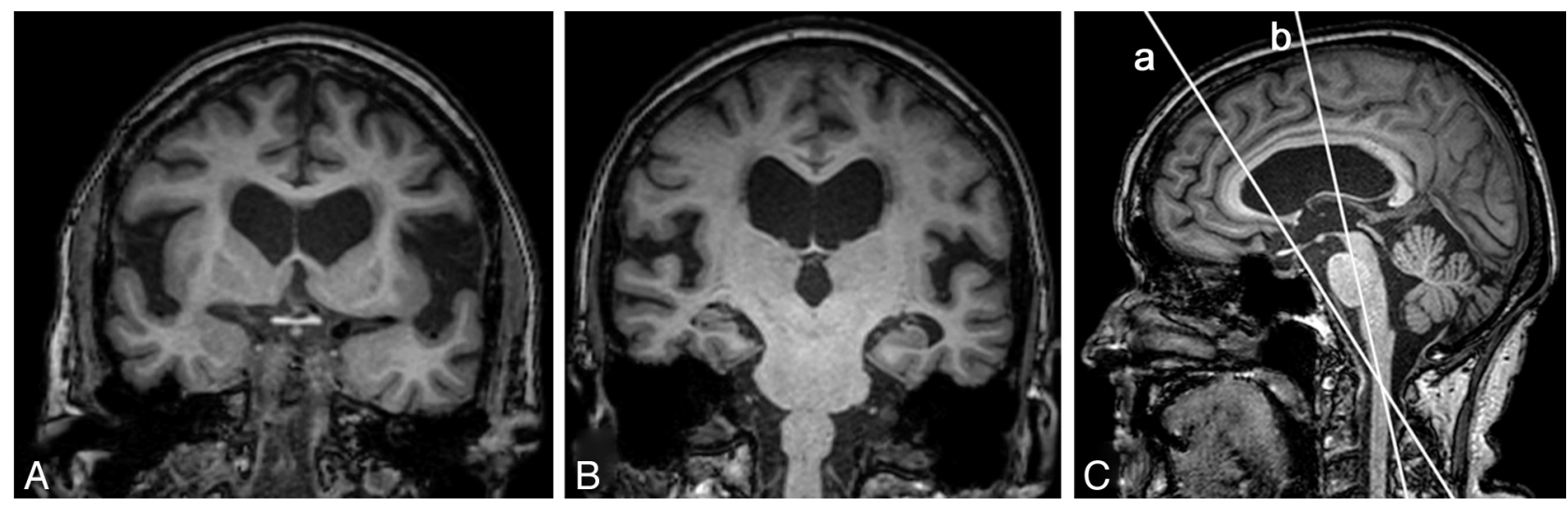

FIG 2. T1-weighted 3D images of a patient with iNPH. $A$ and $B$, Coronal images illustrate the different heights of the Sylvian fissure that can be achieved depending on the angulation of the section. $B$, The Sylvian fissure ordinal. $C$, Sagittal image with orientation lines represented by the coronal images $A$ and $B$.

Table 1: Interrater reliability between 2 independent investigators for all imaging findings

\begin{tabular}{|c|c|}
\hline Imaging Feature & Reliability \\
\hline Evans index (ICC) & 0.93 \\
\hline Callosal angle (ICC) & 0.95 \\
\hline Narrow sulci $(\kappa)$ & 0.64 \\
\hline Sylvian fissure original $(\kappa)^{a}$ & 0.36 \\
\hline Sylvian fissure ordinal $(\kappa)^{b}$ & 0.62 \\
\hline Sylvian fissure height (ICC) (mm) & 0.89 \\
\hline $\mathrm{DESH}^{\mathrm{c}}$ & NA \\
\hline Flow void $(\kappa)$ & 0.33 \\
\hline Focal bulging $(\kappa)$ & 0.28 \\
\hline Third ventricle (ICC) & 0.96 \\
\hline Temporal horns (ICC) & 0.80 \\
\hline DWMH $(\kappa)$ & 0.67 \\
\hline $\mathrm{PVH}(\kappa)$ & 0.72 \\
\hline Focally enlarged sulci ( $\kappa$ ) & 0.54 \\
\hline Aqueductal stenosis $(\kappa)$ & 0.32 \\
\hline
\end{tabular}

Note:-NA indicates not applicable.

a The original method to measure the Sylvian fissure. ${ }^{7}$

b Ordinal scale of $0-2$.

${ }^{c}$ Calculated from Sylvian fissure ordinal and narrow sulci.

stenosis was evaluated on sagittal and coronal images and graded as present or not present.

\section{Statistics}

ICC and $\kappa$ were used to test for reliability between 2 investigators. Univariate associations and associations adjusted for sex, between shunt outcomes, and all imaging features were assessed with logistic regression models. Imaging markers significant in the univariate analyses were adjusted for age, sex, and previous stroke in multiple logistic regression models. Results from the logistic regression models were presented in a forest plot as estimated ORs with a $95 \%$ CI of 1 SD increase for continuous variables and a 1 unit increase for dichotomous and ordinal variables. The difference in the proportion of shunt responders or the level of improvement between patients with or without dichotomous imaging findings was tested with the $\chi^{2}$ test and the Mann Whitney $U$ test, respectively. Correlations were tested by using the Spearman rank correlation coefficient $(r)$ or the $\phi$ coefficient when appropriate. The correlations tested were exploratory and were not decided before the study, and the level of significance was set at $P<$ .01 for correlations. For other analyses, the level of significance was set at $P<.05$. No corrections for multiple analyses were done. Analyses were performed by using SPSS Version 21.0, (IBM, Armonk, New York).

\section{RESULTS}

At 12-month follow-up, 82 of the 108 patients (76\%) had improved after shunt surgery. Mean values $\pm \mathrm{SD}$ at preoperative MR imaging were the following: Evans index, $0.38 \pm 0.04$; callosal angle, $61 \pm 17^{\circ}$; diameter of the third ventricle, $12 \pm 3 \mathrm{~mm}$; diameter of the temporal horns, $7 \pm 2 \mathrm{~mm}$; and the mean height of the Sylvian fissures, $6 \pm 3 \mathrm{~mm}$. Prevalence and grading of imaging features measured on ordinal or dichotomous scales are presented in Table 2 .

The predictive value of the imaging findings is presented as a sex-adjusted OR in Fig 4. For callosal angle, DESH, and temporal horns, the ORs were significant in the univariate analyses, and when adjusted for sex or when adjusted for sex, age, and previous stroke in the 3 separate multivariate logistic regression models. If only patients without complications $(n=76)$ were included in the logistic regression, the ORs for callosal angle, DESH, and temporal horns were still significant.

There were several correlations among different imaging features at the preoperative MR imaging investigations in the range of $r=0.26-0.68$ (Table 3).

Patients without PVH performed better at baseline in the modified Rankin Scale and in the stride length in the Timed Up and Go Test and $10 \mathrm{~m}$ walk compared with patients with PVH $(P<.05)$. Patients without focal bulging performed better in the walking backward $3 \mathrm{~m}$ test compared with patients with the imaging feature present $(P<.05)$. Patients with normal Sylvian fissures performed better in the modified Rankin Scale $(P<.01)$, balance scale $(P<.01), 10 \mathrm{~m}$ walk, and the walking backward $3 \mathrm{~m}$ test $(P<.05)$ compared with patients with dilated Sylvian fissures. At baseline, Sylvian fissure height (millimeters) correlated with both impaired speed and step length in the walking backward $3 \mathrm{~m}$ test at baseline $(r=0.61, P<.001$ and $r=0.66, P<.0001$, respectively). A smaller callosal angle correlated with more impaired scoring in the balance scale $(r=0.26, P<.01)$, and the Evans index correlated negatively with the Mini-Mental State Examination score $(r=-0.27, P<.01)$. 

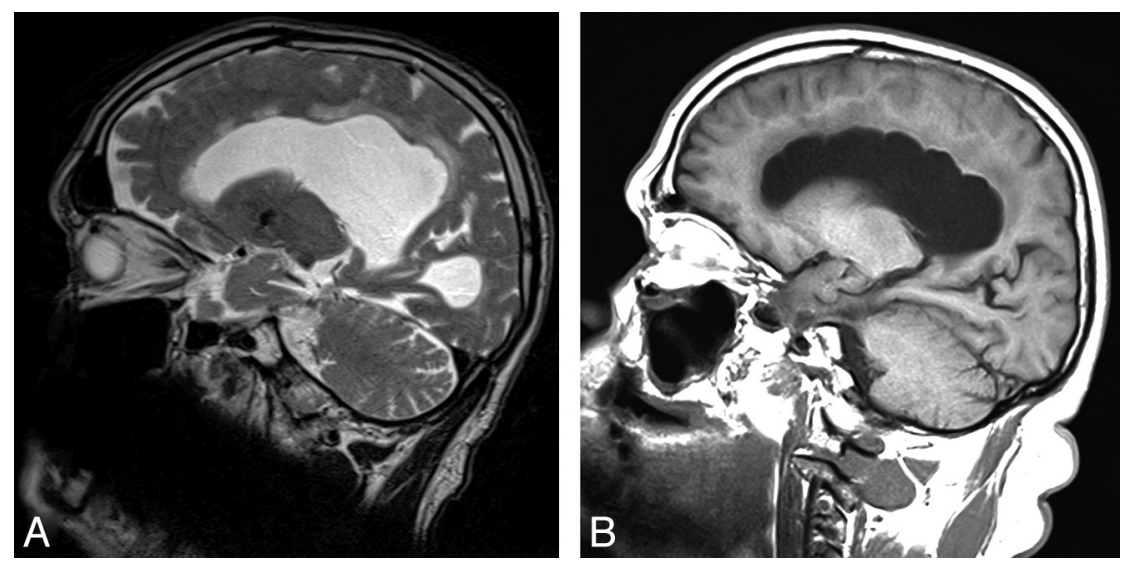

FIG 3. Two patients with focal bulging of the roof of the lateral ventricles. Sagittal images include the most cranial portions of the lateral ventricles. $A$, T2-weighted image. $B$, T1-weighted image.

Table 2: Prevalence and grading of imaging findings measured on dichotomous and ordinal scales

\begin{tabular}{|c|c|c|c|c|c|}
\hline \multirow{2}{*}{$\begin{array}{l}\text { Imaging Feature } \\
\text { (Grading Range) }\end{array}$} & \multicolumn{4}{|c|}{ Frequency of Different Grading (No.) (\%) ${ }^{a}$} & \multirow{2}{*}{$\begin{array}{c}\text { Sample } \\
\text { (No.) }\end{array}$} \\
\hline & 0 & 1 & 2 & 3 & \\
\hline Sylvian fissure ordinal (0-2) & $23(21)$ & $77(71)$ & $8(8)$ & NA & 108 \\
\hline DESH (0-1) & $36(33)$ & $72(67)$ & NA & NA & 108 \\
\hline Flow void $(0-3)^{b}$ & $0(0)$ & $0(0)^{\prime}$ & $9(25)$ & $27(75)$ & 36 \\
\hline Narrow sulci (0-2) & $24(22)$ & $22(20)$ & $62(58)$ & NA & 108 \\
\hline Focal bulging (0-1) & $13(12)$ & $95(88)$ & NA & NA & 108 \\
\hline DWMH $(0-3)$ & $5(5)$ & $11(10)$ & $31(29)$ & $60(56)$ & 107 \\
\hline $\mathrm{PVH}(0-2)$ & $27(25)$ & $66(62)$ & $14(13)$ & NA & 107 \\
\hline Focally enlarged sulci (0-1) & $45(42)$ & $63(58)$ & NA & NA & 108 \\
\hline Aqueductal stenosis (0-1) & $105(97)$ & $3(3)$ & NA & NA & 108 \\
\hline
\end{tabular}

Note:-NA indicates not applicable.

${ }^{a}$ Dichotomous variables: $0=$ not present, $1=$ present.

${ }^{b}$ Flow void in the aqueduct and fourth ventricle.

There was no difference in the proportion of patients with a positive outcome or level of improvement in relation to the Fazekas score among patients with DWMH. The proportion of patients with severe DWMH (Fazekas 3) that improved after shunting was 45/60 (75\%) compared with 36/47 (77\%) in patients with less severe DWMH.

In Table 1, the interrater reliability is presented. For continuous variables, the reliability had the range of $0.80-0.96$ (ICC), and for variables on an ordinal scale, it had a range of $0.33-0.72(\kappa)$. The interrater reliability of dichotomous variables was $0.28-0.67$ (к).

\section{DISCUSSION}

In patients with suspected iNPH, MR imaging is used to aid in the diagnosis and selection of shunt candidates. In this retrospective study of 108 patients with iNPH who had undergone shunt surgery and clinical examination before and at 12 months after surgery, a small callosal angle, occurrence of DESH, and wide temporal horns on the preoperative $\mathrm{MR}$ imaging scans were significant predictors of positive shunt outcome.

A small callosal angle has previously been described as useful for separating patients with iNPH from those with Alzheimer disease and healthy controls. ${ }^{5}$ The results in this and a previous study of a smaller callosal angle in patients who responded to shunt surgery further support its prognostic value. ${ }^{3}$ The significant OR $<1$ for the callosal angle can be interpreted as showing that patients with a larger angle are less likely to benefit from shunt surgery.

Dilation of the temporal horns of the lateral ventricles is an established finding in hydrocephalus ${ }^{13,14}$ and has previously been reported to be more frequent in patients who improve after shunt surgery compared with nonresponders. ${ }^{12}$ This finding was strengthened by the results of the present study. The dilation of the temporal horns in iNPH should not be mistaken for atrophy of the medial temporal lobe as is seen in Alzheimer disease, ${ }^{15}$ even though there are reports of diminished hippocampus volume in patients with NPH. ${ }^{16}$

In the present study, DESH was a predictor of a positive shunt outcome. The imaging feature was discussed in a publication from the Japanese Study of Idiopathic Normal Pressure Hydrocephalus on Neurological Improvement (SINPHONI), and was described as a morphologic hallmark of iNPH. In the SINPHONI study, a positive outcome was achieved in $80 \%$ of 100 patients by using an MR imaging-based diagnostic scheme. ${ }^{8}$ In SINPHONI, 96\% of the patients had dilated Sylvian fissures and could be classified as having DESH compared with $67 \%$ in the present study. Because SINPHONI used narrow supraSylvian subarachnoid spaces as an inclusion criterion in contrast to the present study, the prognostic usefulness of DESH could not be calculated in that study. In CT studies, it has been reported that dilated Sylvian fissures are a sign of poor prognosis, and it has been argued that the dilation is caused by atrophy, which is at odds with the findings in the SINPHONI study. ${ }^{12,17}$ The difference could be explained by the fact that the Sylvian fissure could only be evaluated on transverse images at that time, and it is much easier to visualize the dilation of Sylvian fissures on reconstructed coronal sections as in modern studies. In the present study, the finding of only dilated Sylvian fissures or only narrow sulci at the high convexity did not predict a positive outcome. Nevertheless, in light of our study and the SINPHONI study, it seems as if DESH is a very common finding in iNPH and that it may aid in the selection of shunt candidates.

Several correlations between imaging findings were seen, and some patterns emerged. As expected, there were correlations between markers of the ventricular width and Evans index, and the temporal horns correlated with the diameter of the third ventricle. DESH correlated with a smaller callosal angle and focal bulging of the ventricular roofs.

There was a significant difference at baseline in the severity of motor symptoms and handicap levels between patients with and without PVH, dilated Sylvian fissures, and focal bulging of the ventricular roofs. Correlations were seen between the severity of 


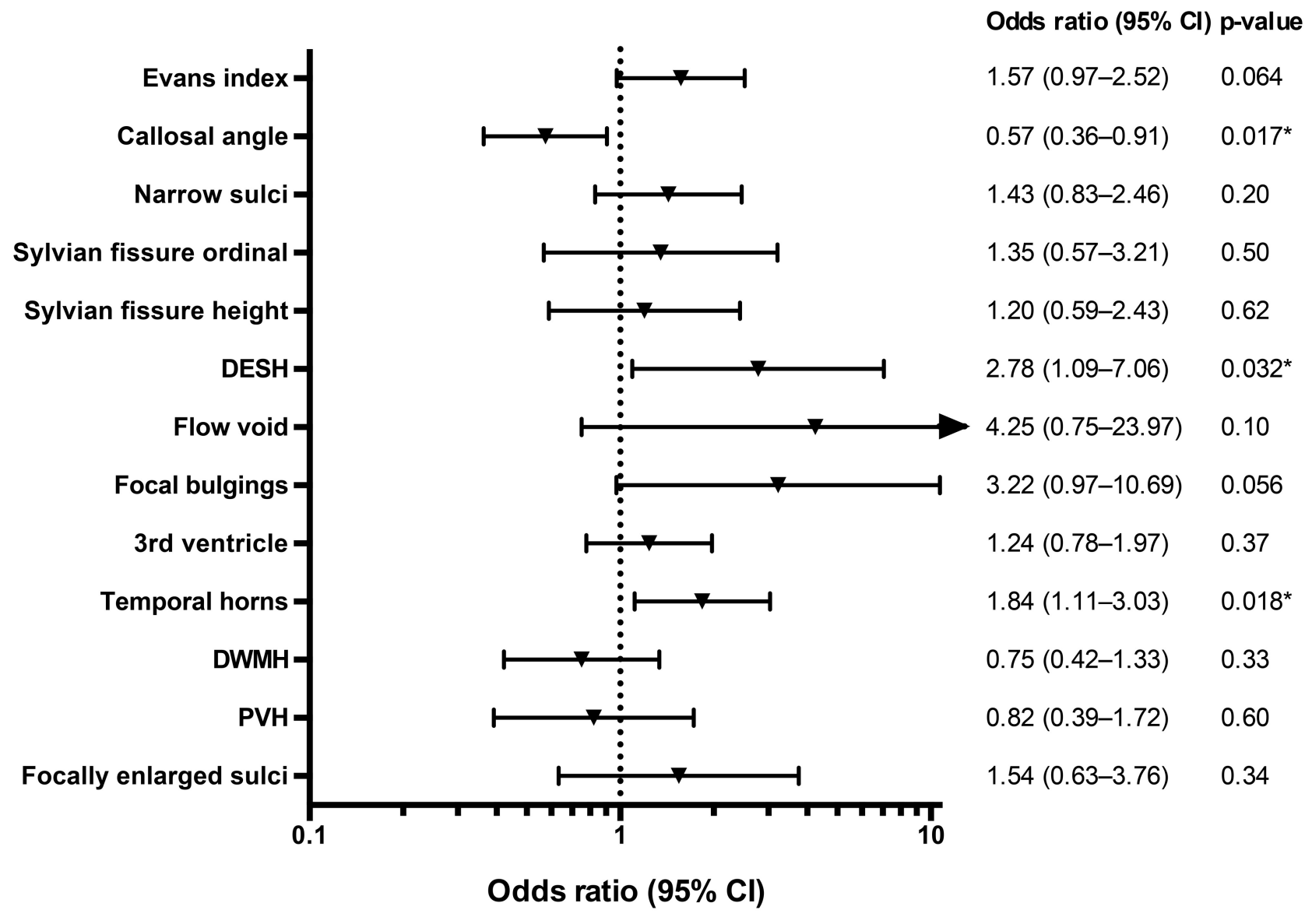

FIG 4. Forest plot with sex-adjusted odds ratios for all imaging features. OR with a $95 \% \mathrm{Cl}$ of $1-S D$ increase for continuous variables and a $1-U$ increase for dichotomous and ordinal variables. An arrow indicates that the confidence interval extends beyond the range of the plot. The Sylvian fissure ordinal is the ordinal scale $0-2$; the Sylvian fissure height is measured in millimeters. The asterisk indicates $P<.05$.

Table 3: Correlations among different imaging markers at baseline $^{\mathrm{a}}$

\begin{tabular}{|c|c|}
\hline Imaging Feature & Significant Correlations \\
\hline Evans index & Third ventricle $\left(r=0.39^{\mathrm{b}}\right)$ \\
\hline Callosal angle & $\begin{array}{l}\mathrm{DESH}\left(r=-0.27^{\mathrm{c}}\right) \text {, focal bulging }\left(r=-0.28^{\mathrm{c}}\right) \text {, } \\
\text { temporal horns }\left(r=-0.33^{\mathrm{d}}\right)\end{array}$ \\
\hline DESH & $\begin{array}{l}\text { Focal bulging }\left(r=0.34^{\mathrm{d}}\right) \text {, focally enlarged sulci } \\
\left(r=0.32^{\mathrm{d}}\right) \text {, callosal angle }\left(r=-0.27^{\mathrm{c}}\right)\end{array}$ \\
\hline Focal bulging & $\begin{array}{l}\text { Temporal horns }\left(r=0.26^{\mathrm{c}}\right) \text {, callosal angle } \\
\left(r=-0.28^{\mathrm{c}}\right), \mathrm{DESH}\left(r=0.34^{\mathrm{d}}\right)\end{array}$ \\
\hline Third ventricle & Temporal horns $\left(r=0.38^{\mathrm{b}}\right)$ \\
\hline DWMH & $\mathrm{PVH}\left(r=0.68^{\mathrm{b}}\right)$ \\
\hline
\end{tabular}

Note:- $r$ indicates Spearman correlation coefficient.

${ }^{a}$ Measurements of Sylvian fissure and narrow sulci at the high convexity were not included because they are components of DESH.

${ }^{\mathrm{b}} \mathrm{P}<.0001$.

${ }^{c} P<.01$.

${ }^{\mathrm{d}} p<.001$.

motor symptoms and a smaller callosal angle or wider Sylvian fissures; and a correlation was seen between a larger Evans index and more impaired cognitive function.

White matter hyperintensities on MR imaging are often divided into $\mathrm{PVH}$, adjacent to the ventricles, and $\mathrm{DWMH}$, located in the deep white matter. Both DWMH and PVH have been reported to be associated with risk factors for vascular disease, such as hypertension and smoking. The origin of the hyperintensities is still debated, and DWMH has been reported to represent gliosis, chronic ischemia, or plasma leakage caused by diffuse cerebrovascular endothelial failure. ${ }^{18}$

In the present study, patients with severe DWMH had a similar positive outcome rate compared with the whole sample, which is in line with the results of Tullberg et al, ${ }^{19}$ who concluded that patients must not be excluded from shunt surgery on the basis of the DWMH findings. In NPH, the PVH around the lateral ventricles is probably caused by transependymal CSF passage into the adjacent white matter, and it has, in some studies, been reported to be a good prognostic sign. ${ }^{20,21}$ We could not replicate that finding, but patients with PVH had more severe gait disturbances and handicap levels at baseline compared with patients without PVH. Because more severe PVH often extends far from the lateral ventricles into the deep white matter, it can sometimes be almost impossible to differentiate PVH from DWMH. This difficulty could be one explanation behind both the high frequency of severe DWMH and the good outcome rate in these patients. Some patients may have had both DWMH and advanced PVH. This possibility is further supported by a strong correlation between DWMH and PVH.

The focal bulging of the roof of the lateral ventricles is an MR imaging feature that, to our knowledge, has not been described previously in iNPH. We do not know the frequency of this finding in other types of ventricular enlargement, obstructive hydrocephalus, or healthy elderly. The focal bulging is best visualized on sagittal images in the section including the most cranial portions 
of the ventricles. The bulging may be caused by CSF pulsations in the lateral ventricles in a weakened spongiotic ependyma and edematous periventricular brain parenchyma, ${ }^{22}$ located between periventricular veins.

The interrater reliability between 2 observers of measures of flow void, focal bulging, and aqueductal stenosis was not satisfactory and neither was the reliability of the original description of the grading of the Sylvian fissure. ${ }^{7,8}$ We showed that the reliability increased from $\kappa=0.36$ to $\kappa=0.62$ by defining the way the sections should be reconstructed and angulated. Dramatic differences in the height of the Sylvian fissure could be obtained depending on which coronal section was used and how it was angulated (Fig 2). All inconsistent grading scores were re-evaluated, and a consensus was reached between the 2 investigators. In accordance with our experience, Ishii et $\mathrm{al}^{5}$ illustrated differences in the callosal angle, depending on the angulation of the coronal section. These differences are important when these imaging features are used in clinical practice, and only well-defined grading scales with high reliability should be used.

In 3 of the cases in this study, there was a subtle focal narrowing of the Sylvian aqueduct. This did not obstruct the CSF flow and was not considered the cause of the hydrocephalus in these patients. It is advisable to evaluate the aqueduct thoroughly in patients with suspected iNPH_-for example, by including a highresolution, heavily $\mathrm{T} 2$-weighted $3 \mathrm{D}$ MR imaging sequence in the protocol to exclude obstructive causes of hydrocephalus.

An interesting finding is the diversity of different morphologic features in this study. All patients had similar symptomatology, but $67 \%$ had DESH, while $10 \%$ had neither dilated Sylvian fissures nor narrow high-convexity sulci; $25 \%$ had no PVH at all, while $13 \%$ had PVH extending all the way to the cortex. Previous studies in iNPH have found both heterogeneous patterns of metabolic disturbances in the cortex measured with FDG-PET and various patterns of $\mathrm{CBF}$ reduction measured with SPECT. ${ }^{23,24}$ These findings raise the question: Are the morphologic differences different stages of the same disease or is iNPH a syndrome of several diseases with different etiologies? There were significant differences in symptom severity between patients with or without $\mathrm{PVH}$, focal bulging, and dilated Sylvian fissures, indicating that these imaging features may be involved in the progression of the disease. However, this question should be addressed in a prospective longitudinal study.

It has recently been discussed that in studies of iNPH, it is important to present descriptive data of the sample, including comorbidity, to facilitate comparisons of different studies. ${ }^{25}$ It seems to be equally important to also describe the radiologic findings in sufficient detail. Otherwise, we cannot know whether we are investigating and performing surgery in similar patients, and comparisons of studies become difficult.

Despite these findings, callosal angle, DESH, or temporal horns cannot be used to exclude patients from surgery. Of patients with a callosal angle of $>90^{\circ}, 4 / 7$ (57\%) improved; 23/36 (64\%) patients without DESH improved; and 7/15 (47\%) patients with temporal horns of $<5 \mathrm{~mm}$ improved from shunt surgery. This illustrates one of the greatest problems with studies of prognostic or nonprognostic investigations in iNPH: The number of patients who do not improve after shunting are too few, even in samples as large as in this study, to draw any conclusions regarding in which patients shunting should not be performed.

Some limitations should be addressed. All patients had been diagnosed with iNPH by a specialized team and had undergone shunt surgery, and the MR imaging investigations used in this study had been used in the clinical evaluation of these patients. We had no control group of healthy individuals or patients with Alzheimer disease. Thus, the results in this study must be interpreted in the light of the highly selected sample, and no conclusions about the diagnostic value of the findings can be made. The clinical radiologic diagnosis was made by several different neuroradiologists without a defined protocol, which might have influenced the frequency of some of the retrospectively evaluated imaging findings. For example, all patients had an Evans index of $>0.30$, and no patient had severe cortical atrophy. However, several of the imaging features investigated in this study were not used clinically at the time of the study at our center. Because of missing sequences in some patients, we could not grade all imaging features in all patients. This gap was most evident for grading of the flow void because we chose to include only investigations from a single scanner to avoid any differences among scanners. ${ }^{26}$ Because of the limited number of patients in whom flow void was graded, not a single patient with a small flow void was found, which probably affected the absence of significant correlations between flow void and other MR imaging findings. Although data were collected prospectively in a standardized way, the radiologic measurements and analyses of clinical data for this study were performed retrospectively. This feature limited the possibility of making corrections when specific MR imaging sequences or some clinical data were missing.

\section{CONCLUSIONS}

The callosal angle, diameter of the temporal horns, and presence of DESH were predictors of a positive outcome after shunting in this sample of patients with iNPH. We recommend that these noninvasive imaging features be assessed in the preoperative evaluation of patients with iNPH because they may aid in the selection of shunt candidates.

\section{ACKNOWLEDGMENTS}

We thank Lisa Wernroth for statistical consultation and the staff of our NPH team at Uppsala University Hospital for their commitment to all the patients.

Disclosures: Johan Virhammar-RELATED: Grant: Selanders Stiftelse,* Comments: Selanders Stiftelse is an independent foundation in Sweden. The institution received a grant to support the cost of this study. Katarina Laurell—RELATED: Grant: Selanders Stiftelse. * Money paid to the institution.

\section{REFERENCES}

1. Adams RD, Fisher CM, Hakim S, et al. Symptomatic occult hydrocephalus with "normal" cerebrospinal-fluid pressure: a treatable syndrome. N Engl J Med 1965;273:117-26

2. Klinge P, Hellstrom P, Tans J, et al. One-year outcome in the European multicentre study on iNPH. Acta Neurol Scand 2012;126: 145-53

3. Virhammar J, Laurell K, Cesarini KG, et al. The callosal angle measured on MRI as a predictor of outcome in idiopathic normal-pressure hydrocephalus. J Neurosurg 2014;120:178-84

AJNR Am J Neuroradiol 35:2311-18 Dec 2014 www.ajnr.org 
4. Hellström P, Klinge $P$, Tans J, et al. A new scale for assessment of severity and outcome in iNPH. Acta Neurol Scand 2012;126:229-37

5. Ishii K, Kanda T, Harada A, et al. Clinical impact of the callosal angle in the diagnosis of idiopathic normal pressure hydrocephalus. Eur Radiol 2008;18:2678-83

6. Sasaki M, Honda S, Yuasa T, et al. Narrow CSF space at high convexity and high midline areas in idiopathic normal pressure hydrocephalus detected by axial and coronal MRI. Neuroradiology 2008;50:117-22

7. Kitagaki H, Mori E, Ishii K, et al. CSF spaces in idiopathic normal pressure hydrocephalus: morphology and volumetry. AJNR Am J Neuroradiol 1998;19:1277-84

8. Hashimoto M, Ishikawa M, Mori E, et al. Diagnosis of idiopathic normal pressure hydrocephalus is supported by MRI-based scheme: a prospective cohort study. Cerebrospinal Fluid Res 2010; $7: 18$

9. Algin O, Hakyemez B, Taskapilioglu O, et al. Morphologic features and flow void phenomenon in normal pressure hydrocephalus and other dementias: are they really significant? Acad Radiol 2009;16: 1373-80

10. Fazekas F, Chawluk JB, Alavi A, et al. MR signal abnormalities at $\mathbf{1 . 5}$ $\mathrm{T}$ in Alzheimer's dementia and normal aging. AJR Am J Roentgenol 1987;149:351-56

11. Holodny AI, George AE, de Leon MJ, et al. Focal dilation and paradoxical collapse of cortical fissures and sulci in patients with normal-pressure hydrocephalus. J Neurosurg 1998;89:742-47

12. Wikkelsö C, Andersson H, Blomstrand C, et al. Computed tomography of the brain in the diagnosis of and prognosis in normal pressure hydrocephalus. Neuroradiology 1989;31:160-65

13. Svendsen P, Duru O. Visibility of the temporal horns on computed tomography. Neuroradiology 1981;21:139-44

14. Tans JT. Differentiation of normal pressure hydrocephalus and cerebral atrophy by computed tomography and spinal infusion test. J Neurol 1979;222:109-18

15. Scheltens P, Leys D, Barkhof F, et al. Atrophy of medial temporal lobes on MRI in "probable" Alzheimer's disease and normal ageing: diagnostic value and neuropsychological correlates. $J \mathrm{Neu}$ rol Neurosurg Psychiatry 1992;55:967-72

16. Golomb J, de Leon MJ, George AE, et al. Hippocampal atrophy cor- relates with severe cognitive impairment in elderly patients with suspected normal pressure hydrocephalus. J Neurol Neurosurg Psychiatry 1994;57:590-93

17. Benzel EC, Pelletier AL, Levy PG. Communicating hydrocephalus in adults: prediction of outcome after ventricular shunting procedures. Neurosurgery 1990;26:655-60

18. Wardlaw JM, Smith C, Dichgans M. Mechanisms of sporadic cerebral small vessel disease: insights from neuroimaging. Lancet Neurol 2013;12:483-97

19. Tullberg M, Jensen C, Ekholm S, et al. Normal pressure hydrocephalus: vascular white matter changes on MR images must not exclude patients from shunt surgery. AJNR Am J Neuroradiol 2001;22:1665-73

20. Poca MA, Mataro M, Del Mar Matarin M, et al. Is the placement of shunts in patients with idiopathic normal-pressure hydrocephalus worth the risk? Results of a study based on continuous monitoring of intracranial pressure. J Neurosurg 2004;100:855-66

21. Børgesen SE, Gjerris F. The predictive value of conductance to outflow of CSF in normal pressure hydrocephalus. Brain 1982;105: 65-86

22. Milhorat TH, Clark RG, Hammock MK, et al. Structural, ultrastructural, and permeability changes in the ependyma and surrounding brain favoring equilibration in progressive hydrocephalus. Arch Neurol 1970;22:397-407

23. Ishii K, Hashimoto M, Hayashida K, et al. A multicenter brain perfusion SPECT study evaluating idiopathic normal-pressure hydrocephalus on neurological improvement. Dement Geriatr Cogn Disord 2011;32:1-10

24. Tedeschi E, Hasselbalch SG, Waldemar G, et al. Heterogeneous cerebral glucose metabolism in normal pressure hydrocephalus. J Neurol Neurosurg Psychiatry 1995;59:608-15

25. Malm J, Graff-Radford NR, Ishikawa M, et al. Influence of comorbidities in idiopathic normal pressure hydrocephalus: research and clinical care-a report of the ISHCSF task force on comorbidities in INPH. Fluids Barriers CNS 2013;10:22

26. Bradley WG Jr, Scalzo D, Queralt J, et al. Normal-pressure hydrocephalus: evaluation with cerebrospinal fluid flow measurements at MR imaging. Radiology 1996;198:523-29 